\title{
Síndrome de Cri du chat: primer reporte en mosaico en el suroccidente colombiano
}

\author{
Cri du chat syndrome: first report of mosaicism \\ in the colombian southwest
}

\author{
María A. Acosta-Aragón1, Marco F. Sierra-Zúñiga²
}

\begin{abstract}
Resumen. El síndrome de Cri du chat es una alteración cromosómica causada por deleciones en el brazo corto de cromosoma 5, las cuales varían en tamaño, desde muy pequeñas que comprometen solo el locus 5p15.2, hasta la pérdida de todo el brazo corto. Las mutaciones se originan de novo en el $80 \%$ a $90 \%$ de los casos. Existen dos regiones críticas para el síndrome de Cri du chat; una ubicada en 5p15.3, cuya deleción se manifiesta con el llanto de maullido de gato y retraso en el habla, y otra ubicada en 5p15.2, cuya deleción se manifiesta como microcefalia, hipertelorismo, retraso psicomotor y mental severo. Se han descrito varios genes implicados localizados en estas regiones críticas; entre ellos, TERT, SEMA5A, CTNND2 y MARCHF6, cuya haploinsuficiencia se asocia con los diferentes fenotipos del Cri du chat. En este artículo se describe el caso clínico de una paciente femenina de 8 meses de vida, con características clínicas y un análisis citogenético en mosaico que confirmaron el síndrome de Cri du chat. Este caso es el primero reportado de esta variante en el suroccidente colombiano.
\end{abstract}

Palabras clave: síndrome del maullido del gato, cromosoma 5, deleción cormosómica, mosaicismo.

Abstract. Cri du chat syndrome is a chromosomal disorder caused by deletions in the short arm of chromosome 5, which vary in size, from very small and involving only the $5 p 15.2$ locus, to the loss of the entire short arm. Mutations originate de novo in $80 \%$ to $90 \%$ of cases. There are two critical regions for Cri du chat syndrome;

\footnotetext{
${ }^{1}$ Médica y Cirujana, MSc en Biología-Genética Clínica, PhD en Genética de Poblaciones Humanas y Genética Forense. Profesora Titular, Departamento de Pediatría, Facultad de Ciencias de la Salud, Universidad del Cauca. Popayán, Colombia. E-mail: morin1924@gmail.com.

${ }^{2}$ Médico y Cirujano, Residente de Pediatría. Departamento de Pediatría, Facultad de Ciencias de la Salud, Universidad del Cauca. Popayán, Colombia.

Conflicto de interés: los autores declaran que no tienen conflicto de interés.

Medicina \& Laboratorio 2020;24:317-324. https://doi.org/10.36384/01232576.339

Recibido el 24 de julio de 2019; aceptado el 21 de noviembre de 2019. Editora Médica Colombiana S.A., 2020
} 
one located at $5 p 15.3$ with a deletion that is manifested as a cat's cry and speech delay, and another located at 5p15.2 with a deletion that manifests as microcephaly, hypertelorism, severe psychomotor and mental retardation. Several involved genes located in these critical regions have been described; among them, TERT, SEMA5A, CTNND2 and MARCHF6, and whose haploinsufficiency is associated with the different phenotypes of Cri du chat. This article describes the clinical case of an 8-monthold female patient, with clinical characteristics and a mosaic cytogenetic analysis that confirmed Cri du chat syndrome. This case is the first reported of this variant in southwestern Colombia.

Keywords: Cri-du-chat syndrome, chromosome 5, chromosome deletion, mosaicism.

\section{Introducción}

El síndrome del Cri du chat o del maullido de gato fue descrito inicialmente por el pediatra y genetista francés Jérôme Lejeune en 1963 [1-4]. Se trata de una alteración cromosómica provocada por deleciones en el brazo corto del cromosoma 5, las cuales varían en tamaño, desde muy pequeñas que comprometen solo el locus 5 p15.2, hasta la pérdida de todo el brazo corto [3-6]. Este síndrome parece ser uno de los síndromes de deleción humana más comunes que predomina en el género femenino, con una incidencia que varía entre 1 en 20.000 a 1 en 50.000 nacimientos $[1,3,4,7]$.

Aunque $80 \%$ a $90 \%$ de las deleciones surgen como mutaciones de novo de origen paterno, posiblemente derivados de la ruptura cromosómica durante la formación de gametos masculinos [6,8], aproximadamente el $10 \%$ a $15 \%$ son el resultado de una translocación parental desequilibrada o de la recombinación de una inversión pericéntrica en uno de los padres [1]. Solo unos pocos casos muestran otras alteraciones citogenéticas raras o menos comunes como mosaicismo $(1,4 \%)$, inversiones $(0,5 \%)$ o cromosomas en anillo $(0,5 \%)[6,9]$.
Actualmente se conoce que existen dos regiones cromosómicas, separadas claramente, identificadas como "regiones críticas del Cri du chat", cuya hemicigosis (pérdida de uno de los dos alelos, normalmente presentes en loci autosómicos) se asocia con fenotipos específicos [10]. Una está ubicada en $5 p 15.3$, y su deleción resulta en la manifestación de un llanto similar a un maullido de gato, el cual desaparece en los primeros años de vida, además de un retraso en el habla $[1,10,11] ; y$, la otra, está ubicada en $5 p 15.2$, cuya deleción se manifiesta como microcefalia, cara redonda, hipertelorismo, micrognatia, pliegues epicánticos, orejas bajas, hipotonía, y retraso psicomotor y mental severo [2,10-13] (figura 1).

Los mosaicos (células con al menos 2 genotipos diferentes en un individuo) son raros, y pueden deberse a un error meiótico o postcigótico [14]. Existen tres principales tipos de mosaicismo: 1) somático, que ocurre solo en las células del cuerpo sin incluir la línea germinal; 2) germinal, que ocurre solo en las células germinales o en sus precursores, pero no se encuentra en otra parte del cuerpo; y, 3) mixto (gonadal y somático), que se produce tanto en las células del cuerpo como en la línea germinal [15]. Las manifestaciones clí- 


\section{Llanto muy agudo similar al maullido de un gato}

- Causado por una malformación de la laringe

- Se hace menos notable y desaparece alrededor de los 2 años

\section{Retraso en el habla y transtorno del lenguaje}

\section{Rasgos faciales}

- Microcefalia

- Ojos muy separados con pliegues cutáneos en el canto interno

- Raíz nasal poco diferenciada

- Orejas en una posición baja

\section{Cognición y conducta}

- Retraso en la adquisición de la marcha

- Retraso mental de grado variable

- Dificultades de atención, hiperactividad

y agresividad

\section{Afectación de otros órganos}

- Malformaciones cardiacas

- Tono muscular bajo

-Problemas músculo-esqueléticos

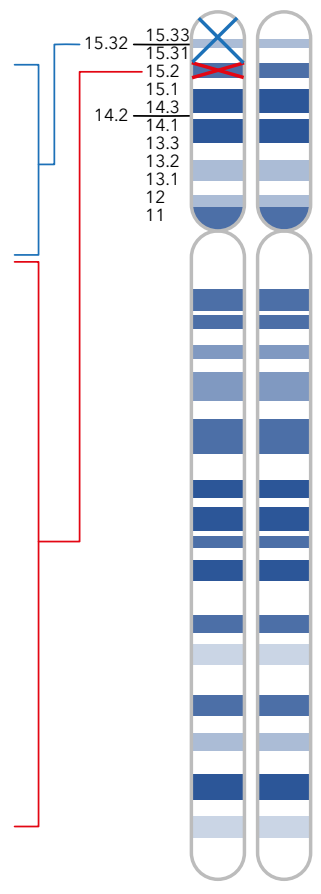

Figura 1. Manifestaciones clínicas del síndrome de Cri du chat, según el lugar de la deleción $5 p$.

nicas en mosaicismos son variables y van a depender del tipo de mutación, el porcentaje de células con la mutación y la distribución del tejido con el cambio genético [15]. El caso que se va a presentar constituye el primero descrito en el suroccidente colombiano con deleción $5 p$ en mosaico.

\section{Caso clínico}

Paciente femenina de 8 meses de vida, producto del cuarto embarazo de una madre de 22 años (G4P0C1A3) y un padre de 26 años, sin consanguinidad, provenientes de la misma región geográfica. La madre refirió que presentó tres abortos espontáneos previos de la misma unión. Gestación planeada con 5 controles prenatales sin aparentes complicaciones. Percepción de movi- mientos fetales a partir del quinto mes de gestación, STORCH negativo, ecografías normales. El embarazo transcurrió en una zona rural del municipio de Cajibío, departamento del Cauca, Colombia. Refirió exposición a agentes químicos tipo herbicidas y fungicidas desde el primer trimestre del embarazo. Parto por cesárea en hospital de tercer nivel a las 35 semanas, por monitoreo fetal no satisfactorio. Peso del neonato $2.120 \mathrm{~g}$, talla y perímetro cefálico no recordados por la madre.

El 8 de agosto de 2017, en el momento de su nacimiento, presentó necesidad de adaptación neonatal asistida, por lo que la paciente estuvo internada en cuidado intensivo neonatal durante 4 meses. Durante su hospitalización en el primer mes de vida, requirió ventilación mecánica invasiva; además, ame- 
ritó tratamiento para crisis convulsivas, ictericia neonatal, falla cardiaca y displasia broncopulmonar. Se documentó un síndrome dismórfico. En evaluación por Genética Clínica se observó al examen físico: peso $2.800 \mathrm{~g}$ con <percentil $(P)_{1},-6$ desviaciones estándar (DE), talla $52 \mathrm{~cm}\left(<\mathrm{P}_{1},-6,6 \mathrm{DE}\right)$. Perímetro cefálico $36 \mathrm{~cm}\left(<\mathrm{P}_{1},-5,23 \mathrm{DE}\right)$, perímetro torácico $34 \mathrm{~cm}\left(<\mathrm{P}_{1}\right)$, perímetro abdominal $32 \mathrm{~cm}\left(<\mathrm{P}_{1}\right)$, distancias intercánticas $70 \times 25 \mathrm{~mm}\left(P_{50}\right)$, filtrum 10 $\mathrm{mm}$, pabellones auriculares $3 \times 2,3 \mathrm{~cm}$ $\left(<\mathrm{P}_{1}\right)$ bilateral, distancia intermamilar $11 \mathrm{~cm}\left(P_{50}\right)$, segmento inferior $19 \mathrm{~cm}$, relación de segmentos 1,84. También se observó microcefalia, cara redonda, hipertelorismo, micrognatia, pabellones auriculares de implantación baja y dismórficos, hipotonía y retraso en su neurodesarrollo (figura 2). Se percibió estridor laríngeo audible a distancia. A la auscultación del tórax, se reportaron roncus en ambos campos pulmonares. Corazón rítmico con soplo holosistólico grado II/VI. Abdomen con botón de gastrostomía sin signos de infección local, peristaltismo lento, y a la palpa- ción era blando, depresible, sin masas ni megalias, ni signos de irritación peritoneal. No se evidenció llanto de maullido de gato.

Se solicitó cariotipo con bandeo $G$ de alta resolución, el cual reportó: 46,XX, del(5)(p15.3)[31]/46,XX[19], es decir, un cariotipo femenino anormal. En el $62 \%$ de las metafases analizadas, se observó una alteración estructural de tipo deleción, en uno de los cromosomas 5, en mosaico. El restante 38\% de las metafases analizadas, mostró un cariotipo normal. Basados en este resultado, se realizó el diagnóstico de síndrome de Cri du chat.

El servicio de Cardiología Pediátrica realizó ecocardiograma, donde se encontró una comunicación interventricular (CIV) perimembranosa subaórtica de $8 \mathrm{~mm}$ con mecanismo parcial de cierre, que dejaba dos orificios, uno apical de $3,3 \mathrm{~mm}$ y otro proximal de 2,2 mm; y una comunicación interauricular ( $\mathrm{ClA}$ ) tipo ostium secundum (OS) de $4 \mathrm{~mm}$. El 12 de diciembre de 2017
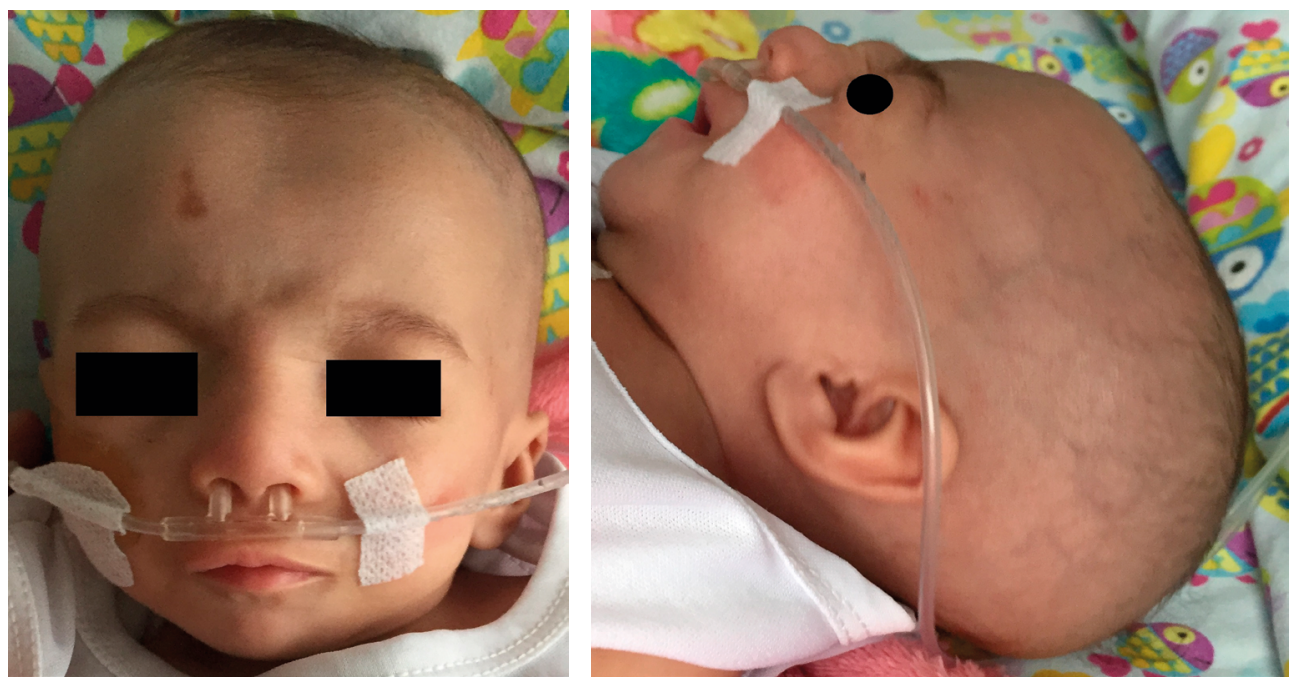

Figura 2. Fotografías de la paciente con microcefalia, cara redonda, hipertelorismo, micrognatia, y pabellones auriculares de implantación baja y dismórficos. 
egresa de la unidad de cuidado intensivo neonatal, con necesidad de oxígeno suplementario domiciliario. El 18 de enero de 2018, consultó nuevamente a centro asistencial por aumento de la dificultad respiratoria. Se documentó una neumonía multilobar y una traqueomalacia, con estancia en unidad de cuidado intensivo pediátrico por riesgo de falla ventilatoria. Se solicitó seguimiento por el servicio de Neumología. La paciente fue dada de alta en condiciones estables.

\section{Discusión}

Este artículo describe el primer caso clínico con síndrome de Cri du chat en mosaico en el suroccidente colombiano, mostrando dos líneas celulares; en el $68 \%$ de las células una deleción $5 p 15.3$, y en el 38\% restante, células normales. La mayoría de las alteraciones genéticas son debidas a deleciones o translocaciones, pero solo unas pocas, como en el caso de esta paciente, muestran otras alteraciones citogenéticas como mosaicismo (1,4\%) [6]. Se determinó un grado de mosaicismo alto, ya que se observó en más del $30 \%$ de las metafases.

Usualmente se cree que el mosaicismo para las aberraciones estructurales es poco común, lo cual se apoya en la literatura mundial $[10,16,17]$. Perfumo y colaboradores en el año 2000, informaron de tres niños con reordenamientos en mosaico $5 p$, dos con una línea celular monosómica parcial y una línea celular monosómica/trisómica parcial, y uno con dos líneas celulares monosómicas parciales diferentes. La frecuencia de mosaicismo que ellos reportaron fue de 3,75\% [1,10]; sin embargo, los datos disponibles sobre reordenamientos estructurales cromosómicos en estudios postnatales y pre- natales reportaron una frecuencia de mosaicismo entre $0,008 \%$ a $0,09 \%$, respectivamente. Se cree que la frecuencia real del mosaicismo podría subestimarse debido al pequeño número de metafases analizadas, que usualmente es de 20. Por el hecho de que algunos pacientes no presentan características fenotípicas anormales, la mayoría permanecen sin detectarse a lo largo de la vida $[10,18,19]$.

Muchos investigadores han descrito las alteraciones cromosómicas y moleculares involucradas en las manifestaciones fenotípicas y clínicas del Cri du chat, entre estos se destacan Overhauser y colaboradores, quienes en 1994 analizaron los puntos de deleción de brazo corto del cromosoma 5 en 49 individuos. Las comparaciones entre las deleciones presentes en los pacientes y sus características clínicas, identificaron varias regiones cromosómicas que estaban involucradas en la expresión fenotípica. Una región cromosómica involucrada en el llanto agudo fue la proximal $5 p 15.3$, mientras que la región cromosómica central 5p15.2 estaba involucrada en las características restantes del síndrome. Las deleciones que no incluyeron estas 2 regiones cromosómicas presentaron diversos fenotipos clínicos, desde retraso mental grave y microcefalia, hasta un fenotipo clínicamente normal [1,11]. En 2003, Zhang y colaboradores [17] estudiaron 10 pacientes con síndrome de Cri du chat, encontrando una deleción del gen TERT que los llevó a concluir que su haploinsuficiencia dificulta el mantenimiento de los telómeros in vivo. El gen TERT codifica para la telomerasa transcriptasa inversa (TERT), una enzima que se encarga de agregar nuevos segmentos de ADN al extremo del cromosoma a medida que la célula se divide, para evitar el acortamiento de los telómeros, el envejecimiento y la apop- 
tosis celular. Está localizado en la porción distal del cromosoma 5 (5p15.33) $[1,4,20,21]$.

En 2001, Cerruti-Mainardi y colaboradores analizaron 80 pacientes con el síndrome, de los cuales 62 tenían una deleción terminal $5 p$ desde p13 a p15.2; siete una deleción intersticial $5 p$; cuatro una translocación de novo; tres una translocación familiar; tres una anomalía $5 p$ de novo que implicaba 2 líneas celulares reorganizadas, y uno tenía una deleción $5 p$ derivada de una inversión paterna. La correlación genotipo-fenotipo en 62 pacientes con deleciones terminales destacó una relación entre la gravedad de la manifestación clínica y el retraso psicomotor, con el tamaño de la deleción [8]. Moreira y colaboradores describieron en 2008 un caso de una niña de 10 años que presentó deleción terminal 5p15.3 en mosaico con una línea celular normal, y quien no manifestó el llanto característico de maullido de gato al momento de su nacimiento, sino un llanto débil pero no agudo, por lo que se concluyó que a pesar de la pérdida de la región crítica del Cri du chat, la presencia de una línea celular normal podía contribuir al fenotipo leve [22]. Según lo anterior, el grado de mosaicismo alto podría también explicar el hecho de que nuestra paciente no presentara llanto de maullido de gato.

También se han descrito otros genes implicados en el síndrome de Cri du chat, como el gen SEMA5A. Su haploinsuficiencia puede interrumpir el desarrollo normal del cerebro y podría conducir a desórdenes del espectro autista, a un retraso en el desarrollo, o a discapacidad intelectual y/o microcefalia [6]. Otro gen implicado es el CTNND2, el cual está asociado con retraso mental severo en el síndrome de Cri du chat $[12,23]$. Se encarga de codificar proteínas neuronales específicas, potencialmente involucradas en el desarrollo del cerebro [13]. Finalmente, se ha descrito también que el gen MARCHF6 puede desempeñar un papel en el llanto agudo de los individuos con deleción 5p [20]. MARCH6 se expresa en el cuero cabelludo y los tejidos torácicos, y también se ha observado en el cerebro, cuello, sistema músculo-esquelético y la glándula suprarrenal [20]. Esta variabilidad en las deleciones $5 p$ puede contribuir a los diferentes fenotipos observados en estos pacientes.

El análisis citogenético proporciona el diagnóstico definitivo en la mayoría de los casos. Si los resultados del estudio citogenético son de apariencia normal o no corresponden con la clínica del paciente, es necesario hacer un análisis citogenético molecular más específico, como hibridación genómica comparativa $(\mathrm{CGH})$, reacción en cadena de polimerasa (PCR) o fluorescencia por hibridación in situ (FISH) [5]. Actualmente, el tratamiento incluye los programas de rehabilitación y manejo integral del paciente, los cuales deben iniciarse tan pronto como sea posible, garantizando así una mejor calidad de vida $[5,7,12,24]$.

\section{Aspectos éticos}

Para la publicación de este caso clíni$\mathrm{co}$, en conjunto con sus fotografías, se obtuvo consentimiento informado de la madre, quien autorizó su divulgación con fines académicos.

\section{Agradecimientos}

A la Universidad del Cauca y su Departamento de Pediatría (Popayán, Colombia). 


\section{Referencias}

1. Kniffin CL, McKusick VA. Cri-du-chat syndrome. Baltimore, Maryland: Online Mendelian Inheritance in Man (OMIM), Johns Hopkins University; 1994. 20 de abril de 2009. Acceso enero de 2019. Disponible en https://www.omim. org/entry/123450\#editHistory.

2. Lejeune J, Lafourcade J, Berger R, Vialatte J, Boeswillwald $\mathbf{M}$, Seringe $\mathbf{P}$, et al. Trois cas de délétion partielle du bras court d'un chromosome 5. C R Acad Sci 1963;257:3098-3102.

3. Honjo RS, Mello CB, Pimenta LS, Nuñes-Vaca EC, Benedetto LM, Khoury RB, et al. Cri du chat syndrome: Characteristics of 73 Brazilian patients. J Intellect Disabil Res 2018;62:467473. https://doi.org/10.1111/jir.12476.

4. Santana-Hernández EE, Tamayo-Chang VJ, Bruzón-Hernández M. Síndrome de $5 p$ menos. Presentación de un caso. MediSur 2014;12:650-654.

5. Hong JH, Lee HY, Lim MK, Kim MY, Kang $\mathbf{Y H}$, Lee $\mathbf{K H}$, et al. Brain stem hypoplasia associated with Cri-du-chat syndrome. Korean J Radiol 2013;14:960-962. https://doi. org/10.3348/kjr.2013.14.6.960.

6. Nguyen JM, Qualmann KJ, Okashah R, Reilly A, Alexeyev MF, Campbell DJ. $5 p$ deletions: Current knowledge and future directions. Am J Med Genet C Semin Med Genet 2015;169:224238. https://doi.org/10.1002/ajmg.c.31444.

7. Galo BL, Alvarenga RH. Síndrome de Cri du chat, una rara cromosopatía. Rev Med Hondur 2012;80:17-19.

8. Cerruti-Mainardi P, Perfumo C, Calì A, Coucourde G, Pastore G, Cavani S, et al. Clinical and molecular characterisation of 80 patients with $5 p$ deletion: genotype-phenotype correlation. J Med Genet 2001;38:151-158. https:// doi.org/10.1136/jmg.38.3.151.

9. Rodríguez-Caballero $A$, Torres-Lagares $D$, Rodríguez-Pérez A, Serrera-Figallo MA, Hernández-Guisado JM, Machuca-Portillo G. Cri du chat syndrome: a critical review. Med Oral Patol Oral Cir Bucal 2010;15:e473-478. https://doi.org/10.4317/medoral.15.e473.

10. Perfumo $C$, Cerruti-Mainardi $P$, Calí A, Coucourde G, Zara F, Cavani S, et al. The first three mosaic cri du chat syndrome patients with two rearranged cell lines. Journal Med Genet 2000;37:967-972. https://doi.org/10.1136/ jmg.37.12.967.

11. Overhauser J, Huang $X$, Gersh $M$, Wilson W, McMahon J, Bengtsson U, et al. Molecular and phenotypic mapping of the short arm of chromosome 5: sublocalization of the critical region for the cri-du-chat syndrome. Hum Mol Genet 1994;3:247-252. https://doi.org/10.1093/ hmg/3.2.247.

12. Gersh M, Goodart SA, Pasztor LM, Harris DJ, Weiss L, Overhauser J. Evidence for a distinct region causing a cat-like cry in patients with $5 p$ deletions. Am J Hum Genet 1995;56:1404.

13. Naumova OY, Rychkov SY, Kuznetzova TV, Odintsova VV, Kornilov SA, Grigorenko EL. DNA methylation alterations in the genome of a toddler with cri-du-chat syndrome. Clin Case Rep 2018;6:14-17. https://doi.org/10.1002/ ccr3.1274.

14. Romano $C$, Ragusa RM, Scillato F, Greco D, Amato G, Barletta C. Phenotypic and phoniatric findings in mosaic Cri du chat syndrome. Am J Med Genet 1991;39:391-395. https://doi. org/10.1002/ajmg.1320390405.

15. Spinner NB, Conlin LK. Mosaicism and clinical genetics. Am J Med Genet C Semin Med Genet 2014;166:397-405. https://doi. org/10.1002/ajmg.c.31421.

16. Niebuhr E. The Cri du chat syndrome: epidemiology, cytogenetics, and clinical features. Hum Genet 1978;44:227-275. https://doi. org/10.1007/bf00394291.

17. Zhang A, Zheng $C$, Hou M, Lindvall $C$, Li KJ, Erlandsson F, et al. Deletion of the telomerase reverse transcriptase gene and haploinsufficiency of telomere maintenance in Cri du chat syndrome. Am J Hum Genet 2003;72:940-948. https://doi.org/10.1086/374565.

18. Kleczkowska A, Fryns JP, Van den Berghe H. On the variable effect of mosaic normal/ balanced chromosomal rearrangements in man. J Med Genet 1990;27:505-507. https:// doi.org/10.1136/jmg.27.8.505.

19. Hsu LY, Yu MT, Richkind KE, Van Dyke DL, Crandall BF, Saxe DF, et al. Incidence and significance of chromosome mosaicism involving an autosomal structural abnormality diagnosed prenatally through amniocentesis: a 
collaborative study. Prenat Diagn 1996;16:1-28. https://doi.org/10.1002/(SICI)10970223(199601)16:1<1::AID-PD816>3.0.CO;2-W.

20. Wu Q, Niebuhr E, Yang $H$, Hansen L. Determination of the 'critical region' for cat-like cry of Cri-du-chat syndrome and analysis of candidate genes by quantitative PCR. Eur J Hum Genet 2005;13:475-485. https://doi. org/10.1038/sj.ejhg.5201345.

\section{Chen CP, Fu CH, Chern SR, Wu PS, Su JW, Lee}

CC, et al. De novo unbalanced translocation resulting in monosomy for distal $5 p$ ( $5 p 14.1 \rightarrow$ pter) and $14 \mathrm{q}(14 \mathrm{q} 32.31 \rightarrow \mathrm{qter})$ associated with fetal nuchal edema, microcephaly, intrauterine growth restriction, and single umbilical artery: Prenatal diagnosis and molecular cytogenetic character- ization. Taiwan J Obstet Gyne 2013;52:401-406. https://doi.org/10.1016/j.tjog.2013.06.005.

22. Moreira LM, de Carvalho AF, Borja AL, Pinto PS, Silveira A, de Freitas LM, et al. Mosaic cridu-chat syndrome in a girl with a mild phenotype. J Appl Genet 2008;49:415-420.

23. Medina M, Marinescu RC, Overhauser J, Kosik KS. Hemizygosity of delta-catenin (CTNND2) is associated with severe mental retardation in cridu-chat syndrome. Genomics 2000;63:157-164. https://doi.org/10.1006/geno.1999.6090.

24. Church DM, Bengtsson U, Nielsen KV, Wasmuth JJ, Niebuhr E. Molecular definition of deletions of different segments of distal $5 p$ that result in distinct phenotypic features. Am J Hum Genet 1995;56:1162-1172. 